\title{
Probes Design and Experimental Measurement of Acoustic Radiation Resistance
}

\author{
Xiaoqing Wang \\ Mechanical Engineering Department, The University of Alabama, Tuscaloosa, AL 35487, USA. \\ Energy and Power Engineering Department, Wuhan University of Technology, Wuhan 430063, China.
}

\author{
Yang Xiang \\ Energy and Power Engineering Department, Wuhan University of Technology, Wuhan 430063, China
}

(Received 5 September 2016; accepted 31 January 2017)

\begin{abstract}
An experimental method used to measure radiation resistance was developed, which was based on a lumped parameter model. Three probes (devices for measuring acoustical radiation resistance) were designed, and the test system was built. To verify the accuracy of the experimental results and to find the application frequencies range for each of the probes, self-resistance and cross-resistance measurements of baffled circular pistons were conducted. The results show that this method is a practical way to obtain the acoustical resistance and that the probe with a $57 \mathrm{~mm}$ speaker can obtain resistance values in the frequency range from $260 \mathrm{~Hz}$ to $1700 \mathrm{~Hz}$. The probe with a $50 \mathrm{~mm}$ speaker, on the other hand, presents resistance in the frequency range from $460 \mathrm{~Hz}$ to $1900 \mathrm{~Hz}$; the range of $700 \mathrm{~Hz}$ to $2600 \mathrm{~Hz}$ is for the probe with a $40 \mathrm{~mm}$ loudspeaker. To verify the actual application effects, experiments measuring the resistance of a horn were performed. The results show that the measuring system with three probes can be used to predict acoustical resistance of various structures with high accuracy in a convenient and simple way.
\end{abstract}

\section{INTRODUCTION}

Active or passive noise control methods ${ }^{1-3}$ are common ways to lower the level of sound power radiated from various products. Designing a quiet structure and considering the mechanical vibration and acoustic characteristics in the design stage of the product is the fundamental method to optimize the radiation resistance of a structure based on the theory of a lumped parameter model, which was developed by Koopmann et al. ${ }^{4-6}$ The resistance matrix of the structure can be obtained by analytical and numerical methods, which are suitable for products with simple boundary conditions., ${ }^{2,7}$ There are still accounts of acoustic boundary value problems that are difficult to compute with the numerical method for the complexity of the geometrical shape. ${ }^{8,9}$ The singularity problem has not been totally solved, although some progress had been made. ${ }^{10-13}$ However, the experimental method was not be affected by this, but it also had the potential to give precise values of the radiation resistance in a frequency ranges. Arenas et al. ${ }^{14-16}$ has studied the experimental method of measuring the acoustic radiation resistance with good results obtained. Even though some studies have been conducted, ${ }^{14-19}$ such as the there is no other research work on the accuracy of the experimental results and the optimal size of the experimental device with its applicable frequency range. The research presented in this paper is aimed to solve these two problems.

\section{THEORY}

\subsection{Lumped Parameter Model}

In a lumped parameter model ${ }^{4,20}$ the acoustic power output of a vibrating product can be written in the resistance matrix with the volume velocities of the surface elements. Each of the surface elements were assumed to be vibrating as pistons. The equation of the power output was determined as

$$
W_{a v}=\frac{1}{2} U^{T} \mathfrak{R} U^{*}
$$

where, $U=\left[U_{1} \cdots U_{N}\right]^{T}$ is the volume velocity of the surface elements and $\mathfrak{R}=\left[\begin{array}{ccc}\mathfrak{R}_{11} & \cdots & \mathfrak{R}_{1 N} \\ \vdots & \ddots & \vdots \\ \mathfrak{R}_{N 1} & \cdots & \mathfrak{R}_{N N}\end{array}\right]$ is the acoustic surface radiation resistance matrix. The resistance matrix could be determined by the numerical method. However, due to the huge amount of preparation work and time-consuming calculations, the experimental method has some advantages in determining the values of the resistance matrix.

\subsection{Resistance Matrix Measurement Theory}

In a lumped parameter model, the single term of resistance matrix is defined as

$$
R_{a, m n}=R\left[\frac{p\left(\vec{r}_{m}\right)}{u\left(\vec{r}_{n}\right)}\right]
$$

where $p\left(\vec{r}_{m}\right)$ is the sound pressure of a point $m$ on the structures surface, and $u\left(\vec{r}_{n}\right)$ is the volume velocity of a point $\mathrm{n}$ on the structure surface. When $m=n$, it is called self- resistance and when $m \neq n$, it is called cross-resistance.

In this experimental method, a small source with a known volume velocity is needed to approximate a point simple source, and it must meet two conditions. First, the size of the source should be small enough so that the influence of its shape can be ignored when it is installed on the structures surface. Second, it must be able to radiate a sound pressure that 
is strong enough to be measured in a certain frequency range, so that every point on the structures surface can be measured. These two requirements are conflicting, because a source with a smaller size means it will has lower power output at low frequency. To simulate a point simple source, ${ }^{21}$ the loudspeaker used must be small compared to the acoustic wavelength, so a flat panel loudspeaker with a small diameter was used in the present study. The loudspeaker was enclosed in a designed cavity to guarantee it is working as a point simple source, which is essential because the loudspeaker will be more representative of a point dipole source if not enclosed. A point dipole source has a very weak radiation output ability at low frequencies. ${ }^{22}$

According to the acoustic theory, the sound pressure inside a small volume cavity is determined by the harmonic oscillation velocity of the cavity wall and it is defined as

$$
\hat{p}=\frac{j \rho_{0} c_{0}^{2}}{\omega V} \hat{u}
$$

where $\rho_{0}$ is the density of the medium, $c_{0}$ is sound velocity in the medium, $\omega$ is the circular frequency $(2 \pi f), V$ is the cavity volume, and $\hat{u}$ is the harmonic oscillation velocity of the cavity wall.

In the analysis, ignoring the influence of a standing wave in the cavity and given the condition that the volume of the cavity and the response of the microphone measuring sound pressure have no phase error, the volume velocity of the chamber wall can be calculated from Eq. (3). In fact, the shape of the loudspeaker is not regular, so it is difficult to measure the volume of the cavity accurately. The microphone responses generally have some phase error. Therefore, it is necessary to calibrate the measurement devices prior to the experiment.

The calibration is conducted with a cavity (called calibration cavity) whose volume is known and fixed on the source cavity. The microphone fixed on the top of the calibration cavity is used to measure the sound pressure in it. Figure 2 below is the schematic diagram for the position of the calibration cavity and the source cavity. The transfer function $F_{c}$, referred to as the sound pressure ratio between the two cavities, is determined by

$$
F_{c}=\frac{\hat{p}_{2}}{\hat{p}_{1}}=\left[\frac{j \rho_{0} c_{0}^{2} \hat{u}_{2}}{\omega V_{2}}\right] /\left[\frac{j \rho_{0} c_{0}^{2} \hat{u}_{1}}{\omega V_{1}}\right] ;
$$

Since the air compression in the source cavity corresponds to the air expansion in the calibration cavity, this means $\hat{u}_{1}=$ $-\hat{u}_{2}$. So the Eq. (4) can be simplified as

$$
F_{c}=\frac{\hat{p}_{2}}{\hat{p}_{1}}=-\frac{V_{1}}{V_{2}}
$$

Thus, $V_{1}$ can be calculated by measuring the sound pressure of the two cavities.

Assuming that the diameter of cavity $V_{2}$ is $d$, the length along the $X$ axis is $l\left(V_{2}=\pi l d^{2} 4\right)$, and the transmission of acoustic wave inside of the cavity is in the form of the plane wave, the sound pressure along the cavity axis ( $z$ axis) can thus be determined by

$$
\hat{p}_{z}=\frac{4 \rho_{0} c_{0}^{2} u}{j \pi d^{2}} \frac{\cos (k(l-z))}{\sin (k l)} ;
$$

where $k$ is the free-field wavenumber. Since $V_{2}=\pi l d^{2} 4$, so Eq. (6) can be simplified as

$$
\hat{p}_{2}=\frac{\rho_{0} c_{0} u}{j \omega V_{2}} \frac{k l}{\sin (k l)} ;
$$

where $\hat{p}_{2}$ is the sound pressure on the top of the cavity $2(z=$ $l$ ). Using $V_{s}$ and $V_{c}$ to represent the volume of the sound cavity and the calibration cavity respectively, and $\hat{p}_{s}$ to represent the sound pressure in the source cavity, gives

$$
\hat{u}=j \frac{\omega V_{c}}{\rho_{0} c_{0}^{2}} \hat{p}_{s} F_{c}
$$

The volume velocity of the arbitrary point $k$ on the surface of the structure can be calculated with Eq. (9)

$$
\hat{u}\left(\vec{r}_{k}\right)=j \frac{\omega V_{c}}{\rho_{0} c_{0}^{2}} \hat{p}_{s}\left(\vec{r}_{k}\right) F_{c} .
$$

When the sound pressure of point $i$ on the surface of the structure is measured, then the acoustic radiation resistance between two arbitrary points can be calculated with

$$
Z_{a, i k}=\mathfrak{R}\left[\frac{p\left(\vec{r}_{i}\right)}{u\left(\vec{r}_{k}\right)}\right]=\mathfrak{R}\left[\frac{\rho_{0} c_{0}^{2}}{j \omega V_{c}} \frac{p\left(\vec{r}_{i}\right)}{p_{s}\left(\vec{r}_{k}\right)} F_{c}^{-1}\right] .
$$

When $i=k$, it is called self-resistance, and if $i \neq k$, it is called cross-resistance.

Then Eq. (10) can be further simplified as

$$
z_{a, i k}=\frac{\rho_{0} c_{0}^{2}}{\omega V_{c}} \operatorname{Im}\left[\frac{p\left(\vec{r}_{i}\right)}{p_{s}\left(\vec{r}_{k}\right)}\right] .
$$

Thus, the acoustic radiation resistance can be calculated with Eq. (11) by amending the phase error of the microphone with $F_{c}^{-1}$ which was calculated by the calibration measurement, and by multiplying the sound pressure of arbitrary points on the surface of the structure and the pressure inside the source cavity with the designed device.

\section{THE MEASUREMENT SET-UP}

A schematic of the instrumentation is shown in Fig. 1. It includes one probe, which is the designed experimental device to measure acoustic radiation resistance and the NI cDAQ-9172 chassis, NI 9263 Analog voltage output module, NI 9234 dynamic signal acquisition modules, and the computer for data acquisition and post-processing program. The loudspeakers are flat panel types and their rated power output is $2 \mathrm{~W}$. In this study, three sets of experimental devices (probes) were designed with the diameters of the three loudspeakers used are $57 \mathrm{~mm}, 50 \mathrm{~mm}$, and $40 \mathrm{~mm}$, respectively and their projected diaphragm diameters are $52 \mathrm{~mm}, 46 \mathrm{~mm}$, and $36 \mathrm{~mm}$, respectively. The name of the microphone is DGO9767CD, and the sensitivities used are $-26 \mathrm{~dB},-30 \mathrm{~dB},-32 \mathrm{~dB}$, and $-50 \mathrm{~dB}$.

The data acquisition program, which was developed by LabVIEW, can gather data automatically after the initial parameters are set up. ${ }^{23}$ The data post-processing program was designed by MATLAB. In each of the experiments, the input volume of the loudspeaker was kept constant the loudspeaker was driven with sinusoidal signals, and the frequency range 


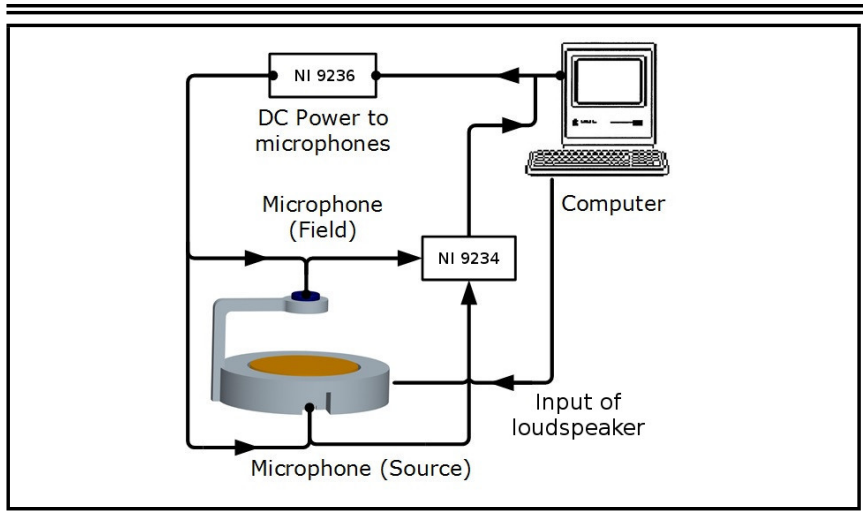

Figure 1. Schematic of experimental set-up.

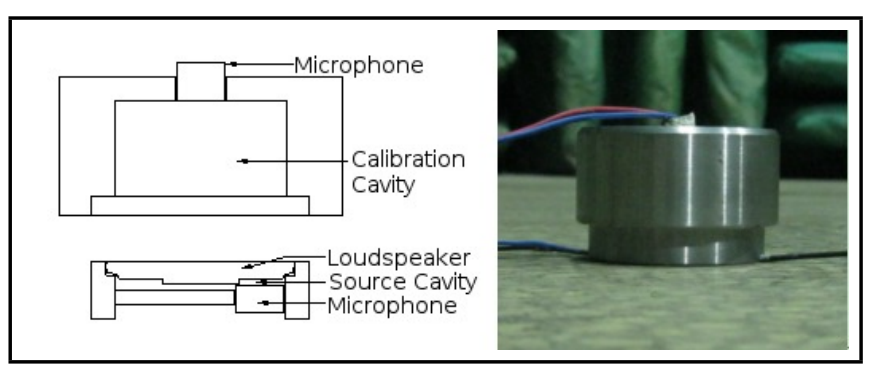

Figure 2. Schematic diagram of the calibration and in-situ experimental setup.

was from 20 to $4000 \mathrm{~Hz}$ with frequency increments of $20 \mathrm{~Hz}$. The sampling rate was $25.6 \mathrm{KHz}$ and the sampling number is 20000. The acoustical radiation resistance was calculated with the actual experimental data and $F_{c}$ calculated by the calibration measurement.

Before the formal measurements, calibration of the experimental devices is needed. As illustrated in Fig. 2, the experimental device was placed on the floor of a hemi-anechoic chamber, which has an acoustical treatment on the walls and ceiling only as well as feature hard floors with no acoustical treatment. ${ }^{24}$ The design of the calibration cavity is based on the calibration principle developed above. Its length is determined by the attenuation of sound pressure along the calibration cavity axis within $1 \mathrm{~dB}$. Thus, the loudspeaker stimulates acoustic fields synchronously in the source cavity and the calibration cavity. The microphone fixed on the top of the calibration cavity measures the sound pressure in the calibration cavity. Based on former studies, ${ }^{25-27}$ after reducing the effect of the major factors influencing the accuracy of the measurement, the results obtained showed greater improvements. Each of the three devices' transfer function between the calibration cavity and the source cavity calculated by calibration measurements is illustrated as in Fig. 3.

Recalling Eq. (5), the transfer function between the pressure in the calibrator and the speaker cavities should be constant as a function of frequency with its imaginary part identically zero. However, it is known that the actual physical systems always have some difference from their idealized models and the difference will lead to the systems having some limitations in their actual application. It can be seen from Fig. 3 that the transfer function will vary with the frequency in the actual measuring conditions, which is caused by the different phase response between the two microphones together with other factors brought by the loudspeaker and the other associated elec- a)

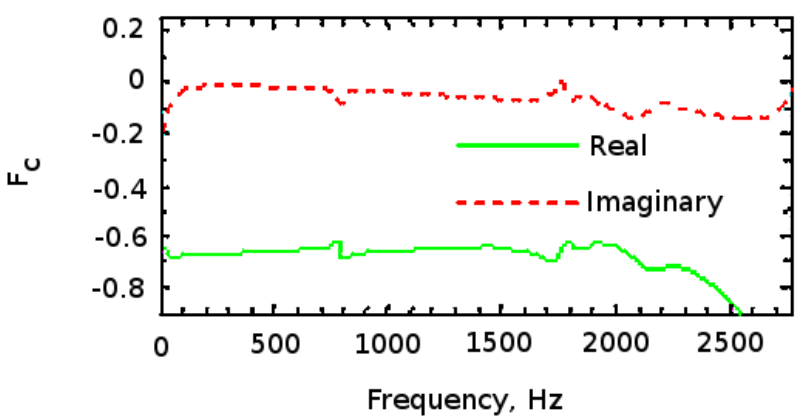

b)

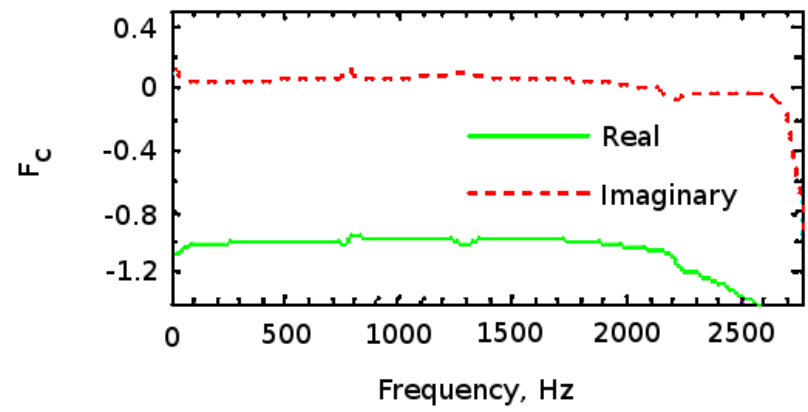

c)

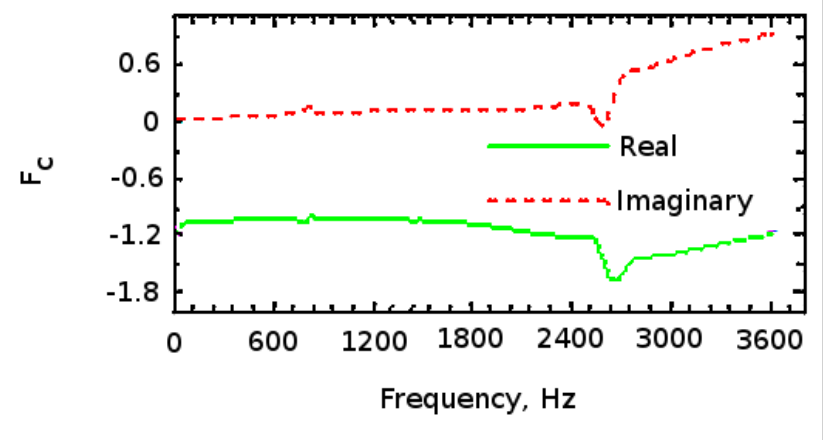

Figure 3. Results of calibration for the three devices: Loudspeaker, $P=2 \mathrm{~W}$, (a) $D=57 \mathrm{~mm}$, (b) $D=50 \mathrm{~mm}$, and (c) $D=40 \mathrm{~mm}$.

tronics, such as capacitors and resistors.

Even though the imaginary parts of transfer functions show some fluctuations, they stay close to zero, tending to be linear variation. The real parts approximation show straight lines in the range of the interested frequencies, which suggests that the loudspeakers have a good frequency characteristic. The stable working range of frequencies for the loudspeakers will become wider with the decreasing of the loudspeaker's diameter.

\section{APPLICABLE FREQUENCY RANGE OF THE PROBE}

Two kinds of tests were conducted to verify the ability of the experimental method determining the acoustical resistance: verification of the self- resistance measurements and verification of cross-resistance measurements. 


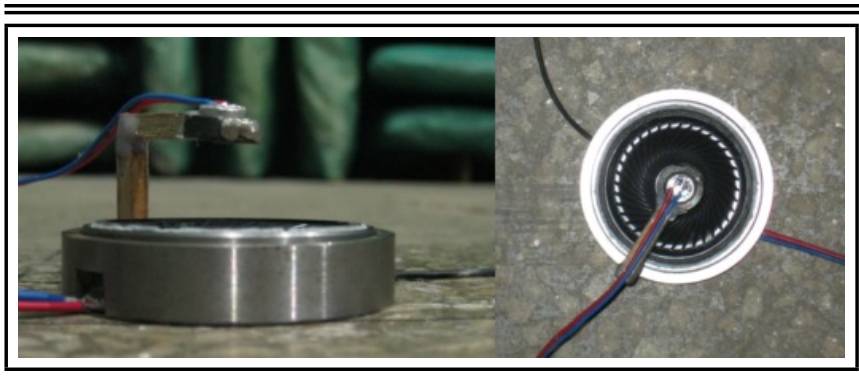

Figure 4. Self-resistance measurement of baffled circular piston.

\subsection{Self-resistance of Baffled Circular Piston}

To simulate measuring the resistance of a piston in an infinite baffled plate, the experimental device was placed on the floor of the hemi-anechoic chamber, as illustrated in Fig. 4. The influences of the device's height were neglected in this study, as they have a relatively low height compared to the size of the hemi-anechoic chamber.

Referring back to Eq. (11), the radiation resistance can be calculated with the experimental data and $F_{c}$. For the convenience of comparison, the experimental solutions were changed to a dimensionless quantity by multiplying the area of the speaker diaphragm $S_{i}$ (below) and then divided by the characteristic impedance of the acoustic medium $\rho c$. By assuming the diaphragm of the loudspeaker moves as a piston, the self-resistance $\mathfrak{R}_{i i}$ of the baffled circular piston was calculated using the Eq. $(12)^{28}$

$$
\mathfrak{R}_{i i}=\rho_{0} c S_{i}\left(1-\frac{J_{1}(2 k r)}{k r}\right) ;
$$

where $J_{1}$ is the first order Bessel function and $r$ is the radius of the loudspeaker's diagram. The measured self-resistances are compared with the analytical values respectively, as shown in Fig. 5.

It can be seen from the comparison in Fig. 5 that the experimental values agree well with the analytical values in a wide frequency range, which states that this experimental method is able to obtain the acoustical resistance. However, there are significant differences at the low frequencies. The experimental results are far from the analytical values and also show some natural random fluctuations, which will lighten with the diaphragm of the loudspeakers increasing from $36 \mathrm{~mm}$ to $52 \mathrm{~mm}$. This phenomenon only occurred at the low frequencies because of the lower SNR (signal-to-noise ratio). At certain frequencies and currents, the smaller the diameter of the loudspeaker's diaphragm, the lower the power radiated from the diaphragm. Thus, the radiation resistance measurement is limited by the noise floor of this hemi-anechoic chamber, which is at approximately $R S \rho c=1 \times 10^{-2}$. Therefore, the experimental results will not show good agreement with the analytical values below 0.01 . That is why the degree of agreement between the experimental and the analytical values declines. The low frequency limitations will ascend at the same time.

In addition, the difference between them becomes bigger at high frequencies, for example, above $2000 \mathrm{~Hz}$, because the condition of the lumped parameter model will not be satisfied there. When the wavelength is shorter, the ratio of it to the size of the probe is not big enough and the measuring probe a)

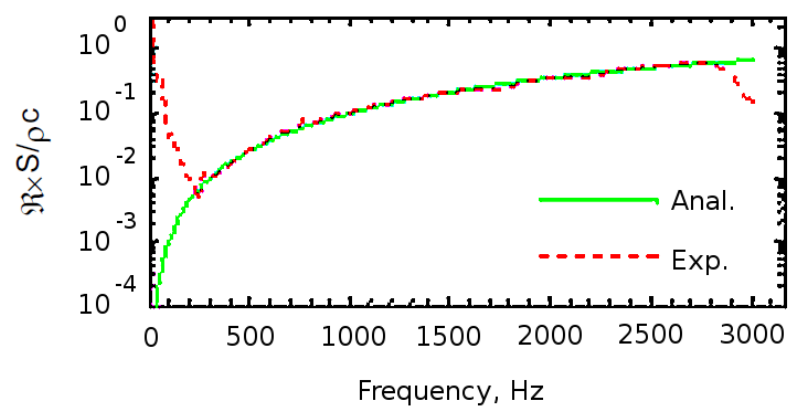

b)

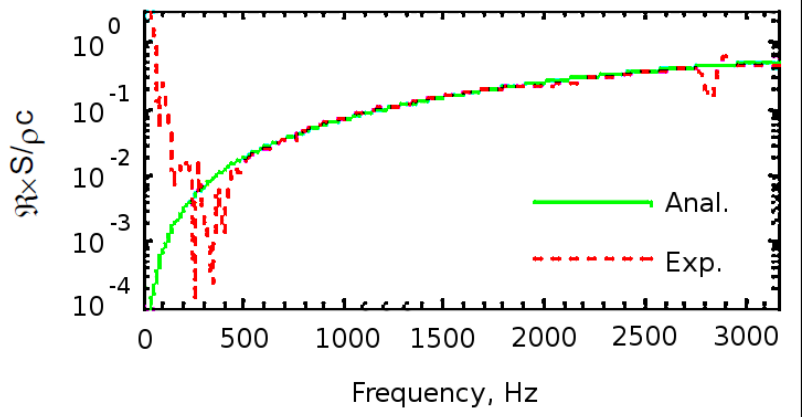

c)

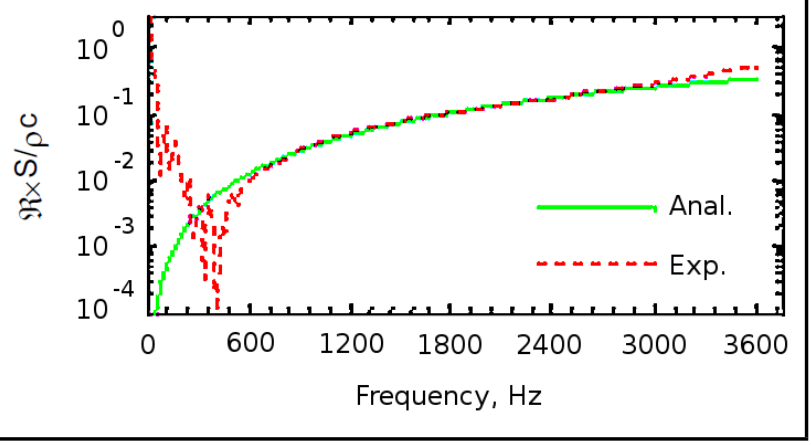

Figure 5. Results for the normalized self-resistance of baffled circular piston: Loudspeaker, $P=2 \mathrm{~W}$, (a) $D=57 \mathrm{~mm}$, (b) $D=50 \mathrm{~mm}$, and (c) $D=$ $40 \mathrm{~mm}$.

should not be viewed as a point simple source. This is not in correspondence with the condition of the lumped parameter model and thus the performance of the measuring probe will be poor.

\subsection{Cross-resistance of Baffled Circular Piston}

As stated in the Eq. (1), it is known that the cross-resistance terms take a large proportion of the resistance matrix in computing the average sound power of the vibration structure. Using the approach of the discrete calculation method, Hashimoto (2001) presented the approximated values of the cross-resistance between the $i$ th element and the $k$ th element, ${ }^{29}$

$$
R_{i k}=\frac{2 \rho_{0} c k^{2} S_{i} S_{k}}{\pi}\left[\frac{J_{1}\left(k r_{i}\right)}{k r_{i}} \frac{J_{1}\left(k r_{k}\right)}{k r_{k}}\right] \frac{\sin k d_{i k}}{k d_{i k}} ;
$$

where $S_{i}$ and $S_{k}$ are the areas of the $i$ th and $k$ th elements respectively, $r_{i}=\sqrt{S_{i} \pi}$ and $r_{k}=\sqrt{S_{k} \pi}$ are the equivalent radii of the $i$ th and $k$ th elements respectively, and $d_{i k}$ is the distance between the centers of the two circular vibrating piston elements. 


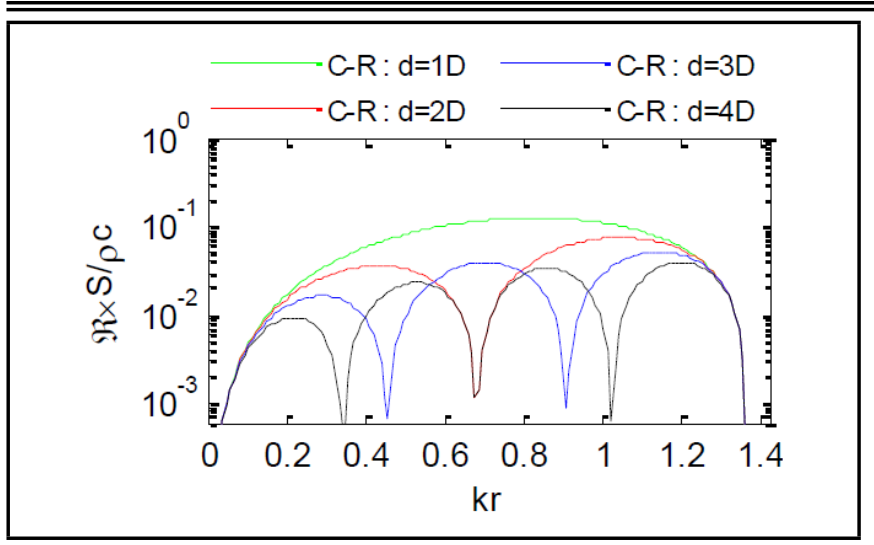

Figure 6. Results of the cross-resistance for two circular elements $\left(r=r_{i}=\right.$ $\left.r_{k}=0.026 \mathrm{~m} ; d=d_{i k}=n D(n=1,2,3,4 ; D=0.057 \mathrm{~m})\right)$.

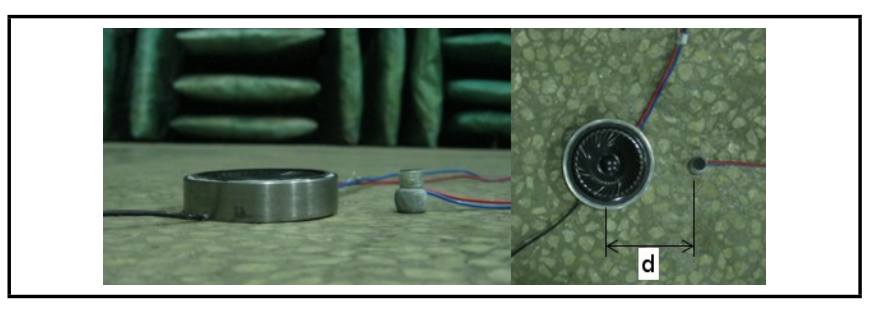

Figure 7. Cross-resistance measurement of baffled circular piston $(d=0.057 \mathrm{~m})$.

Thus, the two circular elements are taken as an example, $r_{i}=r_{k}=0.026 \mathrm{~m}, d_{i k}=n D(n=1,2,3,4 ; D=0.057 \mathrm{~m})$, and the absolute value of cross-resistance (C-R) for the $i$ th and $k$ th elements are presented in Fig. 6 .

It can be seen that the results of the cross-resistance decrease when the distance between the two elements increase. There are more valleys with the increasing of the distance, which will seriously reduce the power radiated from the elements. Thus, only the first 3 steps off- axis cross-resistance of the devices were measured.

Similar to the self-resistance measurements, the device was placed on the floor of the hemi-chamber, and the field point was measured with the microphone at one, two and three $d_{i k}$ $(57 \mathrm{~cm}, 50 \mathrm{~cm}$ and $40 \mathrm{~cm})$ from the center of the loudspeaker. The in-situ cross-resistance measurement of a baffled circular piston is shown in Fig. 7 and the comparison of the experimental and analytical results are shown in Fig. 8.

The results show very good agreement between the experimental and analytical results. The degree of the agreement between the experimental and the analytical results goes down with the diameter of the loudspeaker decreasing from $60 \mathrm{~mm}$ to $40 \mathrm{~mm}$, which resulted from the same reasons mentioned above. The first one is the lower SNR due to the powder radiated from the diaphragm, especially for the speakers with a small diaphragms as the smaller the diaphragm, the lower the powder radiated from it. Another reason is the frequency limitation from the lumped parameter model as the requirements for it will not be satisfied at high frequencies, which lead to the big errors.

Based on the self-resistance and cross-resistance tests, the applicable frequency range can be determined respectively. The device with a loudspeaker of $60 \mathrm{~mm}$ can obtain resistance values that agree well with the analytical solutions in the frequency range from 260 to $1700 \mathrm{~Hz}$, which is almost the same a)

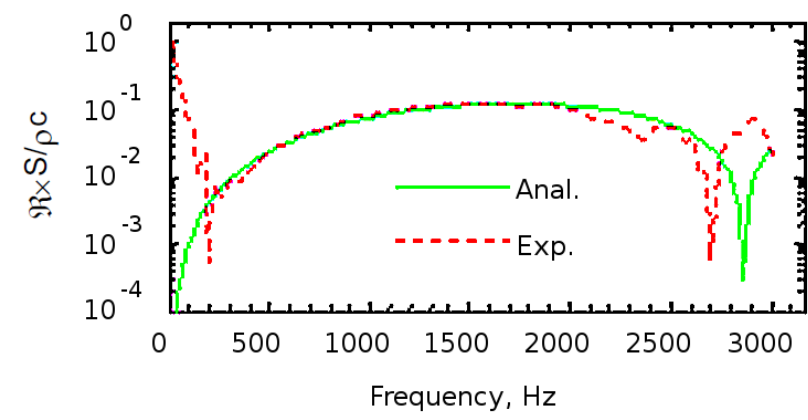

b)

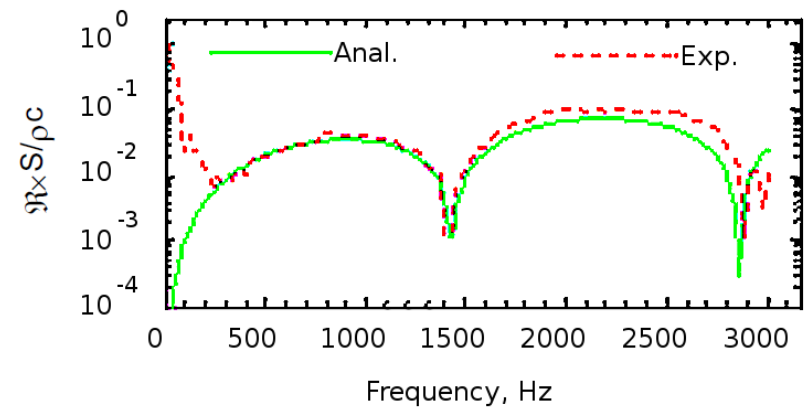

c)

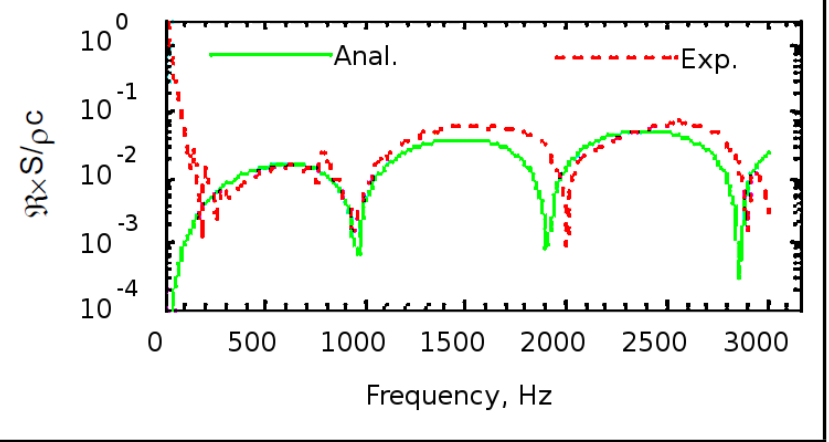

Figure 8. Results of Cross-Resistance Verification (Loudspeaker $D=57 \mathrm{~mm}$, $\left.d_{i k}=57 \mathrm{~mm}\right)$ : (a) One Step Off-Axis $(n=1)$, (b) Two steps off-axis $(n=$ $2)$, and (c) Three steps off-axis $(n=3)$.

linear variation frequency range of the transfer function. The device whose diameter of the loudspeaker is $50 \mathrm{~mm}$ presents a precise resistance value in the frequency range from $460 \mathrm{~Hz}$ to $1900 \mathrm{~Hz}$, and, $700 \mathrm{~Hz}$ to $2600 \mathrm{~Hz}$ for the device with a $40 \mathrm{~mm}$ diameter loudspeaker.

\section{APPLICATION OF THE PROBES IN THE PIPE AND HORN}

The pipe is a critical application scenario for sound transmission. One of its important functions is that it can be used to amplify, silence or even eliminate sound. Take a horn with high power output for daily use as an example. As is shown in Fig. 11, the horn is circular symmetry with its radius varying as a complex function of the distance along the centerline. It is made of $2 \mathrm{~mm}$ aluminum alloy with length of $355 \mathrm{~mm}$ and the radius at the throat and mouth are $128 \mathrm{~mm}$ and $500 \mathrm{~mm}$, respectively. There is a connection tube at the throat of the horn with the same radius as the throat and its length is $40 \mathrm{~mm}$.

During the test, the horn was placed on the floor of a hemianechoic chamber and the gap between it and the floor was 


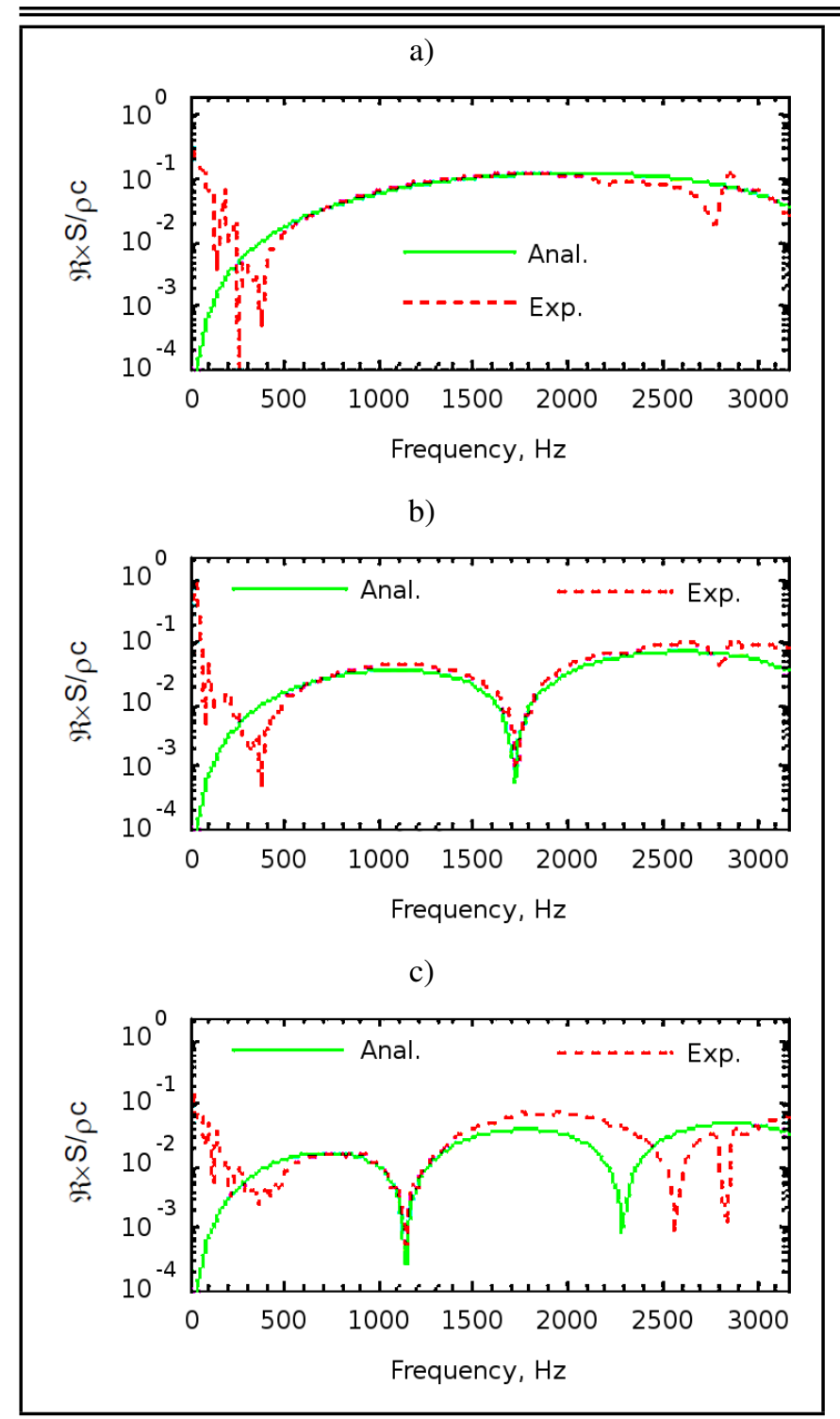

Figure 9. Results of Cross-Resistance Verification, (Loudspeaker $D=$ $50 \mathrm{~mm}, d_{i k}=50 \mathrm{~mm}$ ): (a) One Step Off-Axis $(n=1)$, (b) Two steps off-axis $(n=2)$, and (c) Three steps off-axis $(n=3)$.

sealed by the BLU Tack. The measuring probe was placed at the center of the throat of the horn, as illustrated in Fig. 11.

To verify the accuracy of the measured results, they were compared to numerical values because the cross-section of the horn changes continuously and therefore cannot be analyzed using Webster's horn equation which is applicable to exponential horns, conical horns and so on. ${ }^{15,16}$ The numerical values were calculated with the boundary element method (BEM) using Virtual.Lab.Acoustics, in which the air is set as a homogeneous medium with density and velocity of the sound. The boundary condition of velocity is exerted at the center of the tube's bottom in a circular area with radius same as the diaphragm of the loudspeaker used in the tests. In acoustical grid model, the field point and boundary condition of velocity is shown in Fig. 12. The comparison between the measured results and numerical values of the radiation resistance as a function of frequency is shown in Fig. 13. The frequency increment in both case is $2 \mathrm{~Hz}$.

As can be seen from Fig. 13, the test results agree well with the numerical results which indicates that the designed devices can obtain the resistance of the horn with high accuracy. There a)

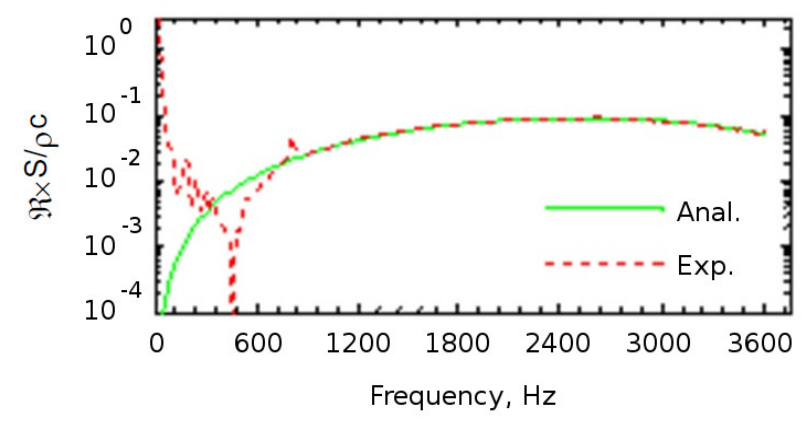

b)

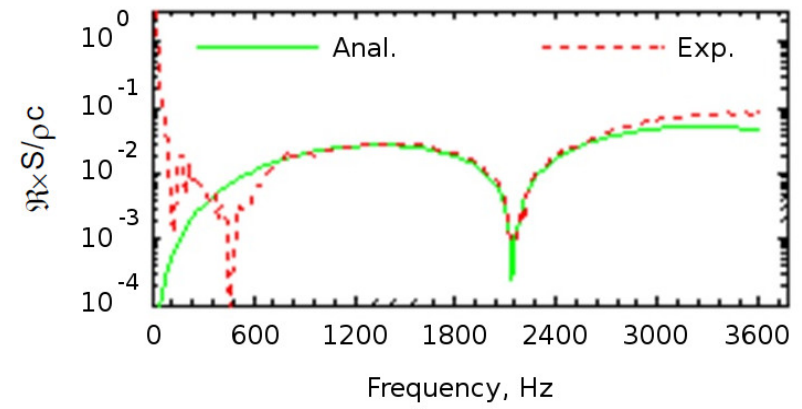

c)

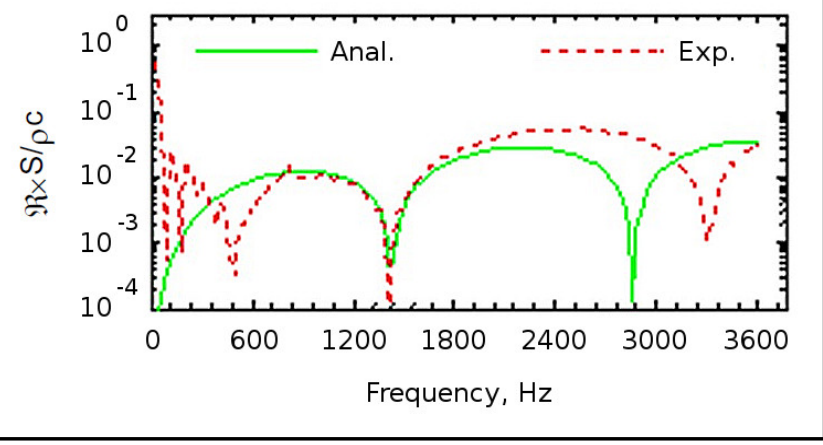

Figure 10. Results of Cross-Resistance Verification, (Loudspeaker $D=$ $40 \mathrm{~mm}, d_{i k}=40 \mathrm{~mm}$ ): (a) One Step Off-Axis $(n=1)$, (b) Two steps off-axis $(n=2)$, and (c) Three steps off-axis $(n=3)$.

are some differences at the low frequencies and high frequencies, which has the same reasons as discussed above. In addition, the inaccuracy of the horn model might also results in lead to the difference between the test and numerical values. There would be some error between the model used in calculation and the actual size of the horn, because the detail size of the horn is not easily measured accurately.

Through this application, it can be seen that the measuring system can present the resistance results with high accuracy. This will save a lot of time in building the model and making calculations, especially when the structures have complex shapes. The system is convenient and very easy to operate, and thus can be used to determine the acoustical resistance matrix of various structures.

\section{CONCLUSIONS}

In this paper, a method has been presented to measure the acoustic radiation resistance. With the three sets of probes designed, the self-resistance and cross-resistance of the infinite baffled piston source had been conducted. Comparing the ex- 


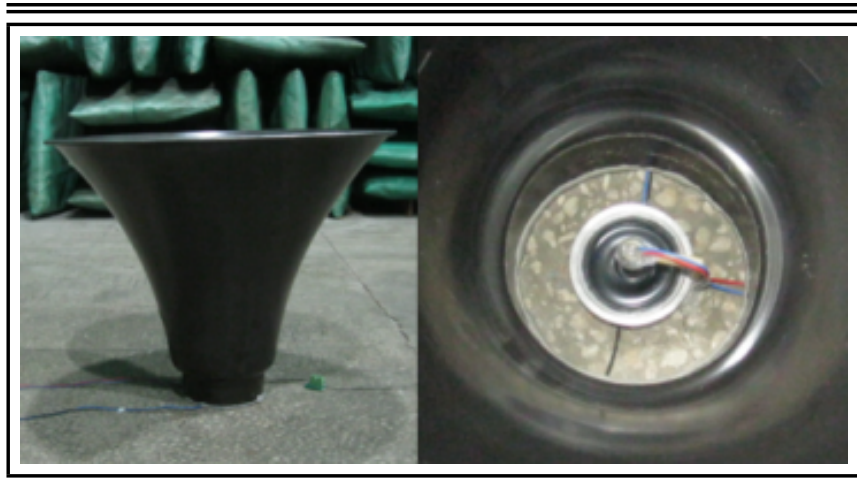

Figure 11. In-situ measurement of horn.

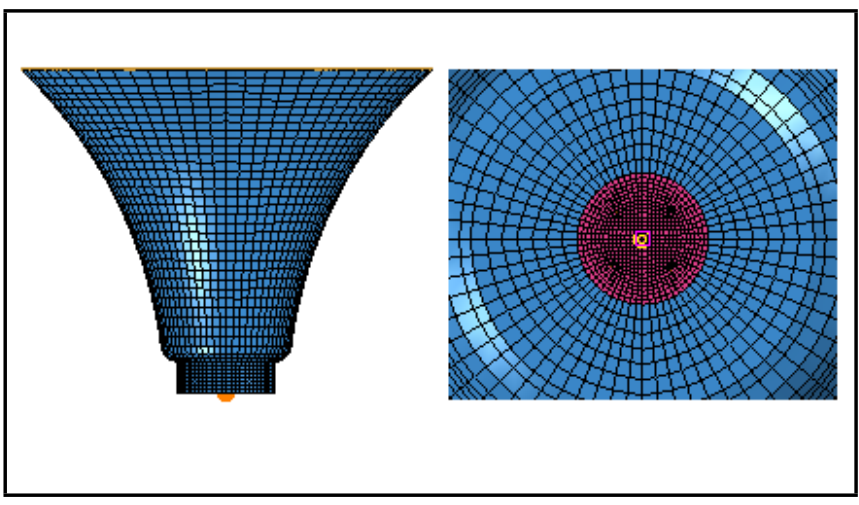

Figure 12. Acoustic grid model of horn.

perimental and analytical results showed that this method is applicable to obtain the acoustical resistance and the applicable frequency ranges for the three devices tested. The frequency ranges $260 \sim 1700 \mathrm{~Hz}, 460 \sim 1900 \mathrm{~Hz}$, and $700 \sim 2600 \mathrm{~Hz}$ for the devices with $57 \mathrm{~mm}, 50 \mathrm{~mm}$, and $40 \mathrm{~mm}$ loudspeakers, respectively were obtained.

Then the resistance at the throat of a horn was tested to verify actual application effects. The results showed that the measuring system with three probes can be used to measure various structures" acoustical resistance with a high accuracy in a convenient and simple manner.

The qualities of the loudspeakers, such as harmonic distortion and characteristic sensitivity, have significant influence on the experimental results. The results would be better at low frequencies if the loudspeaker has a high quality with high power output.

\section{ACKNOWLEDGEMENTS}

This work was supported by the National Natural Science Foundation of China (Grant No. 5107911851279148). Xiaoqing Wang also gratefully acknowledges the help from Xuebao Xia, Yuxiao Shi, Rui Li, Sichong Qian, Lei Shi, and Peng Xue.

\section{REFERENCES}

1 Marburg, S. Developments in structural-acoustic optimization for passive noise control, Archives of computational methods in engineering, 9 (4), 291-370, (2002). https://dx.doi.org/10.1007/bf03041465

2 Liu, Z., and Maury, C. Numerical and experimental study of Near-Field Acoustic Radiation Modes of a baffled a)

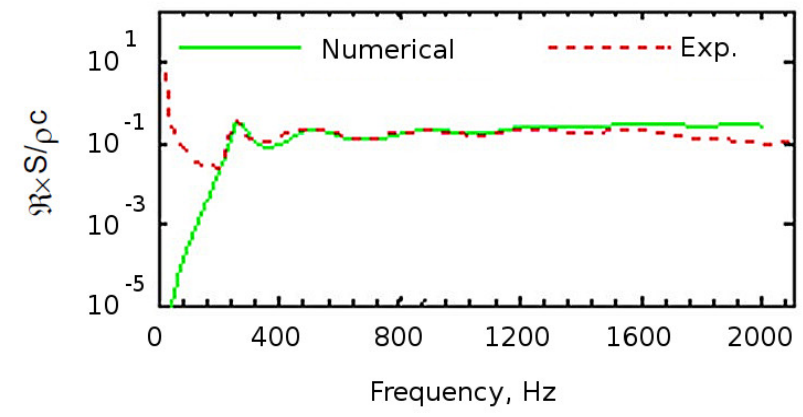

b)

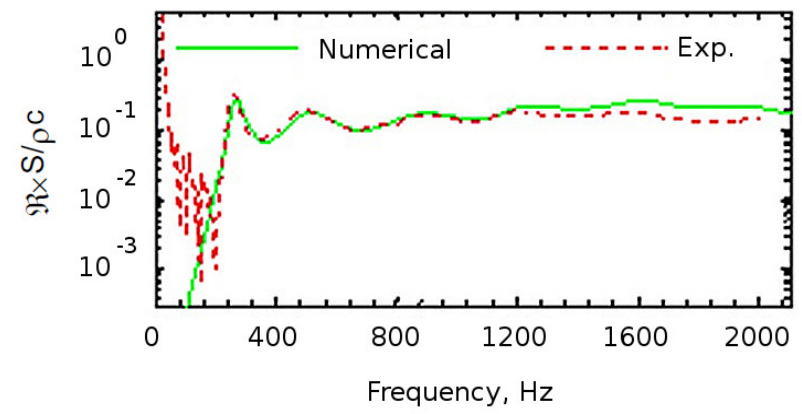

c)

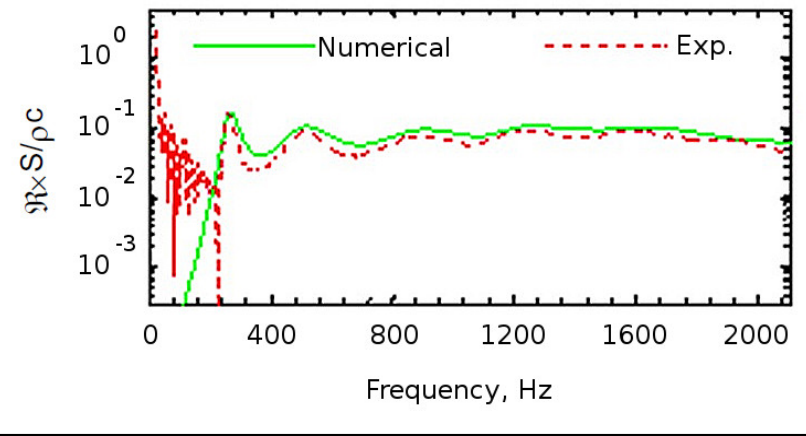

Figure 13. Comparison between the tests results and numerical values of the radiation resistance of the horn: Loudspeaker, $P=2 \mathrm{~W}$, (a) $D=57 \mathrm{~mm}$, (b) $D=50 \mathrm{~mm}$, and (c) $D=40 \mathrm{~mm}$.

spherical cap, Applied Acoustics, 115, 23-31, (2017). https://dx.doi.org/10.1016/j.apacoust.2016.08.010

3 Wise, S., and Leventhall, G. Active noise control as a solution to low frequency noise problems, Journal of Low Frequency Noise, Vibration and Active Control, 29 (2), 129-137, (2010). https://dx.doi.org/10.1260/02630923.29.2.129

${ }^{4}$ Fahnline, J. B., and Koopmann, G. H. A lumped parameter model for the acoustic power output from a vibrating structure, The Journal of the Acoustical Society of America, 100 (6), 3539-3547, (1996). https://dx.doi.org/10.1121/1.417330

5 Xiang, Y., Guo, Z., and Wang, X. Research on design of the resistance probe and experiments using the probe, Journal of Wuhan University of Technology (Transportation Science \& Engineering), 36 (6), 1140-1142, (2012).

6 Xiang, Y., Guo, Z., and Wang, X. Research on the Measurement Principle Design and Calibration of the Resistance 
Probe, Journal of Wuhan University of Technology (Transportation Science \& Engineering), 36 (5), 899-906, (2012).

7 Faverjon, B., and Soize, C. Equivalent acoustic impedance model. Part 2: analytical approximation, Journal of Sound and Vibration, 276 (3-5), 593-613, (2004). https://dx.doi.org/10.1016/j.jsv.2003.08.054

8 Robjohns, H. A brief history of microphones, First published Microphone Data Book, (2001).

9 Crocker, M. J., Arenas, J. P., and Dyamannavar, R. E. Identification of noise sources on a residential splitsystem air-conditioner using sound intensity measurements, Applied Acoustics, 65 (5), 545-558, (2004). https://dx.doi.org/10.1016/j.apacoust.2003.10.008

10 Zellers, B., Koopman, G., Hwang, Y., and Naghshineh, K. Element Free Structural Acoustics for Efficient Shape Optimization, Proc. ASME International Mechanical Engineering Congress and Exposition. https://dx.doi.org/10.1115/imece2005-82958

11 Wu, S., Xiang, Y., Xia, X., and Wang, X. Research on a method for acoustic superposition in element free spatial discrete domains, Journal of Huazhong University of Science and Technology (Natural Science Edition), 41 (11), 22-25, (2014).

12 Xia, X., Xiang, Y., Wang, X., and Wu, S. Research on the acoustic radiation resistance calculation based on wave superposition method, Journal of Ship Mechanics, 19 (1), 206-214, (2015).

13 Qian, S., Xiang, Y., Xiao, X., and Wang, X. Application of Gammatone Filter Bank to Vibration Characteristics Analysis of Engine Cylinder Head, Chinese Internal Combustion Engine Engineering, 34 (6), 36-42, (2013).

14 Arenas, J. P., and Crocker, M. J. Numerical solution for the transmission loss in pipes and reactive expansion chambers of gradual area change, Noise Control Engineering Journal, 49 (5), 224-230, (2001). https://dx.doi.org/10.3397/1.2839664

15 Arenas, J. P., and Crocker, M. J. Approximate Results of Acoustic Impedance for a Cosine-Shaped Horn, Journal of Sound and Vibration, 239 (2), 369-378, (2001). https://dx.doi.org/10.1006/jsvi.2000.3062

16 Arenas, J. P., and Crocker, M. J. A note on a WKB application to a duct of varying cross-section, Applied Mathematics Letters, 14 (6), 667-671, (2001). https://dx.doi.org/10.1016/s0893-9659(01)80024-0

17 Paddock, E. H., and Koopmann, H. G. The Use of Radiation Resistance Measurements to Assess the Noise Characteristics of Machines, Journal of American Society of Mechanical Engineers, 3 (B), 655-662, (1995).
18 Driesch, P. L., Iwata, H., Koopmann, G. H., and Dosch, J. Development and evaluation of a surface acoustic intensity probe, Review of Scientific Instruments, 71 (10), 39473952, (2000). https://dx.doi.org/10.1063/1.1311944

19 Aewnas, J. P., (2001). Analysis of the Acoustic Radiation Resistance and Its Applications to Vibro-Acoustic Problems, Doctor PhD Thesis, Auburn University.

20 Arenas, J. P., Ramis, J., and Alba, J. Far-Field Sound Radiation from a Baffled Elliptical Piston Using a LumpedParameter Model and Singular Value Decomposition, 15th International Congress on Sound and Vibration, Daejeon, Korea, 1633-1640, (2008).

21 Bruneau, M. Fundamentals of acoustics, John Wiley \& Sons, (2013).

22 Oskooi, A. F., Roundy, D., Ibanescu, M., Bermel, P., Joannopoulos, J. D., and Johnson, S. G. MEEP: A flexible free-software package for electromagnetic simulations by the FDTD method, Computer Physics Communications, 181 (3), 687-702, (2010). https://dx.doi.org/10.1016/j.cpc.2009.11.008

23 Wang, X., (2013). Research on the Experimental Measurement and Feasible Technical Solutions of Acoustic Radiation Resistance, Master Master, Wuhan University of Technology Wuhan.

24 Davidson, M. A. Hemi-anechoic chamber qualification and comparison of room qualification standards, The Journal of the Acoustical Society of America, 135 (4),2379-2380, (2014). https://dx.doi.org/10.1121/1.4877859

25 Wang, X., Xiang, Y., Guo, Z., Xia, X., Shi, Y., Xue, $\mathrm{P}$, and $\mathrm{Wu}, \mathrm{S}$. Research on experimental measurement of acoustic resistance and major accuracy influencing factors analysis, J Mech Sci Technol, 28 (4), 1219-1227, (2014). https://dx.doi.org/10.1007/s12206-014-0302-1

26 Wang, X., Xiang, Y., Shi, Y., Wu, S., and Xue, P. Experimental Research on the Measurement of Acoustic Radiation Impedance of Several Typical Acoustic Structures, Journal of Wuhan University of Technology, 35 (1), 151156, (2013).

27 Wang, X., Xiang, Y., Shi, L., Qian, S., and Guo, Z. Design of acoustic radiation resistance measuring system and analysis of factors influencing measuring accuracy, Journal of Applied Acoustics, 33 (3), 228-237, (2014).

28 Pierce, A. D. Acoustics: an introduction to its physical principles and applications, McGraw-Hill New York, (1981).

29 Hashimoto, N. Measurement of sound radiation efficiency by the discrete calculation method, Applied Acoustics, 62, 429-446, (2001). https://dx.doi.org/10.1016/s0003$682 \times(00) 00025-6$ 